\title{
Application of Wi-Fi Wireless Communication Technology in the Remote Monitoring of the Greenhouse
}

\author{
Yunliang Wang ${ }^{1}$, Weisong $\mathrm{Di}^{2}$ \\ ${ }^{1}$ Tianjin Key Laboratory for Control Theory \& Application in Complicated System, Tianjin University \\ of Technology, Tianjin 300384,China; \\ ${ }^{2}$ College of Automation, Tianjin University of Technology, Tianjin 300384, China
}

Keywords: greenhouse, embedded, network communication, remote monitoring

\begin{abstract}
This paper uses the embedded microcontroller processor as the core, introduce a kind of method which used Wi-Fi to increase the distance and simplify the network building method, the GPRS and Internet network communication technology to realize remote transmission of data, and use the web page as the Monitoring interface, realize the acquisition and control of the temperature and humidity of the greenhouse.
\end{abstract}

\section{Introduction}

With the promotion of facility agriculture, greenhouse agriculture in China is developing to intelligent and automation. And the emergence of the Internet of things provided many choices to the researchers.

The traditional method based on cable transmission mode has some disadvantages, such as inconvenient, the wiring is not flexible and not conducive to farming. Push-button greenhouse control system based on PLC can't realize the remote on-line and real-time control ${ }^{[1]}$; by using the method of wireless communication can realize remote monitoring, and avoid the above disadvantages, such as greenhouse monitoring system based on Zigbee ${ }^{[2]}$, but the environment in the greenhouse is complex, it will influence the performance of wireless communication, also the stability of the nodes and its sensors are affected ${ }^{[3]}$, so this article designed the remote monitoring system based on Wi-Fi and GPRS.

The main characteristics of Wi-Fi are high speed, high reliability, can easily combined with the existing wired Ethernet network, and the cost of networking is lower ${ }^{[4]}$. And in recent years low-power Wi-Fi has got rapid development and application, such as CC3000, it has been applied in the high-end washing machine and temperature control system of family.

GPRS transmit data in the form of packet, its cost of data transfer is lower, transmission speed is high $^{[5]}$, so in recent years it was favored by more and more people. Because of its separate for remote monitoring of construction cost and operation cost is higher, this paper combine the short range wireless communication with the long distance wireless communication.

\section{System hardware structure}

In order to realize the remote transmission of data, this system used wireless communication technology and computer network technology, etc, realize the automatically collection of data, remote transmission and the local data store.

The system can be divided into manual control mode and intelligent control mode. In manual control mode, users control the temperature and humidity; in intelligent control mode, users set the temperature and humidity values, and then processed by the data processing center.

The system hardware is composed of three parts: information acquisition and control system, data processing center, user monitoring terminal. The system hardware structure is shown in figure 1 . 


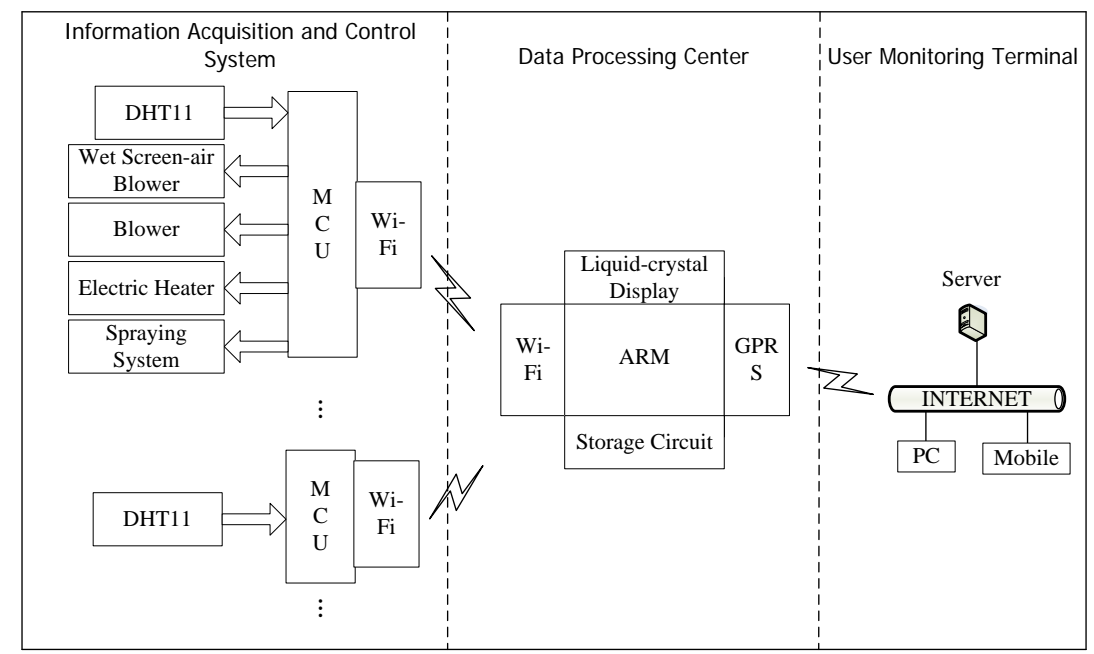

Fig.1 System Hardware Structure

Information acquisition and control system include multiple acquisition nodes and control nodes, it collects the temperature and humidity through DHT11, and uses Wi-Fi module transfer the data to the data processing center.

The data processing center includes Wi-Fi module, liquid-crystal display circuit, storage circuit, and GPRS module. The data processing center analyses and stores the data, then sends it to the user monitoring terminal. And the data processing center also decodes the user commands, according to the control mode send different control instructions. data.

The user monitoring terminal is a computer which has a fixed IP. Set it as a server to receive the

\section{System software design}

System software includes three parts: information acquisition and execute program, data handler program, user monitoring program.

The data processing center receives data and analyses the temperature and humidity respectively through data handler program, and then forwards the data to the user monitoring terminal. Software flow diagram of the system is shown in figure 2.

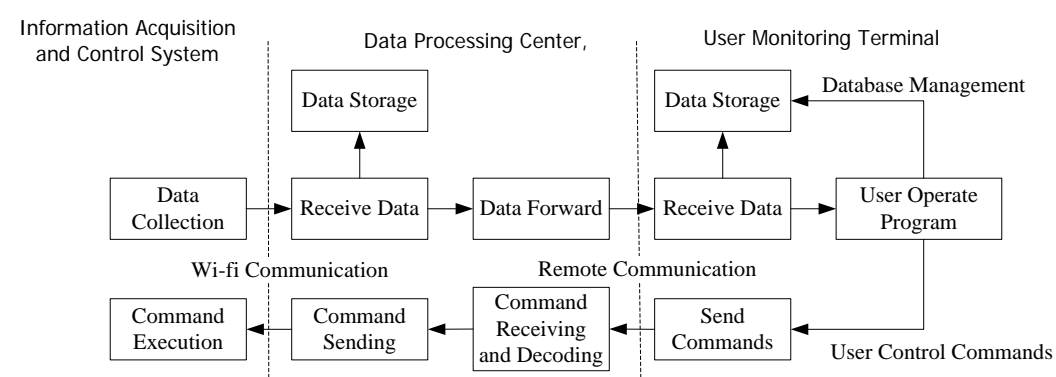

Fig.2 Software flow diagram

The processing and remote transmission of data is mainly completed by data handler program. It analyses the data, decodes user commands, determines the control mode, sends the correct adjustment commands. The flow chart of data processing program is shown in figure 3.

After received the user commands, data processing program decodes them first, then determines control mode. It sends control commands directly in the manual mode; in intelligent control mode, it analyses the commands and sends processed commands.

It should be pointed out that it must call a function to reset the data store when it receives data or sends data through Wi-Fi module to avoid data error.

The user monitoring program is in charge of data receiving and storage, sending user commands, it is the bridge between monitoring interface and data processing center. User monitoring program flow chart is shown in figure 4. 


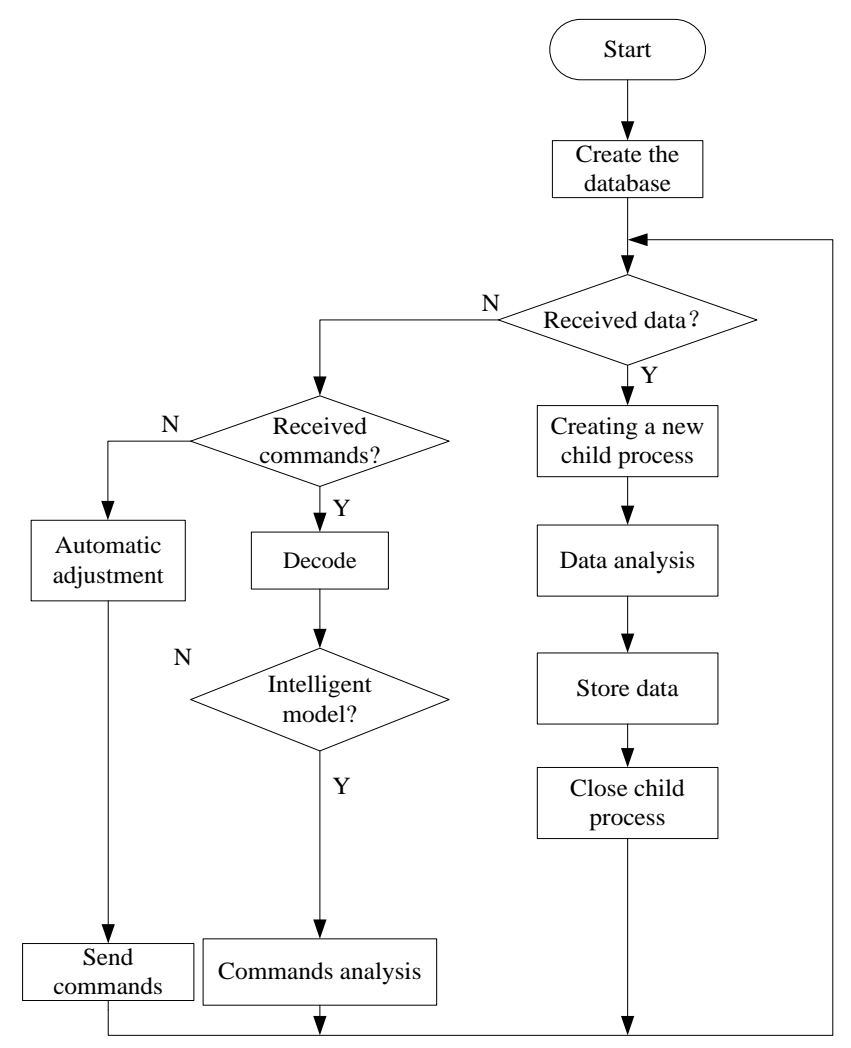

Fig.3 Data processing program flow chart

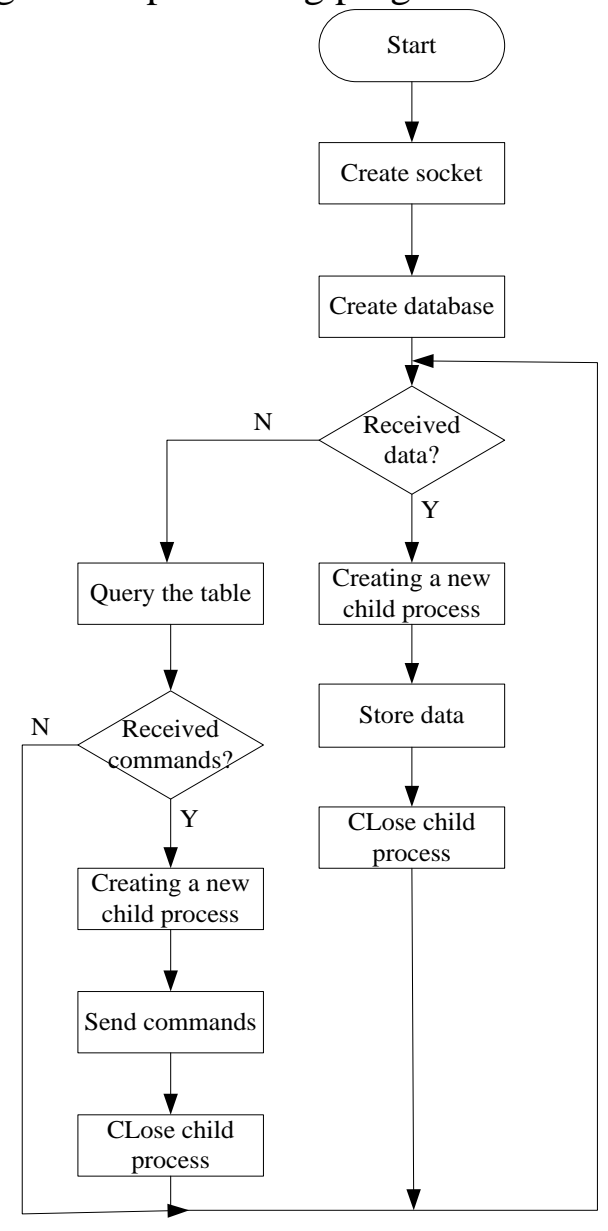

Fig.4 User monitoring program flow chart

In the process of remote communication, the socket played a leading role. The communication between the client and server has three steps: server listen (waiting for the connection, monitoring network request), client request (connect request), connection confirm. Each communication 
program has a socket to describe itself, different processes communicate with each other through its own socket.

The user monitoring terminal installed a database which is sqlite3, it has no independent operation process ${ }^{[6]}$, and its speed and safety are also very prominent. It is important to note that after stored the data or commands, it must call the destructor function to destruct the data struct, in order to avoid memory leaking.

\section{The analysis of system test results}

Because the data processing program and the user monitoring program operate the database, when compile them we can't use gcc or arm-linux-gcc directly, we must specify the path of the necessary library, then transplant the executable file to the ARM. Run the user monitoring program first, then run the data processing program, the test is taken in the laboratory, and the monitoring interface is shown in the figure.

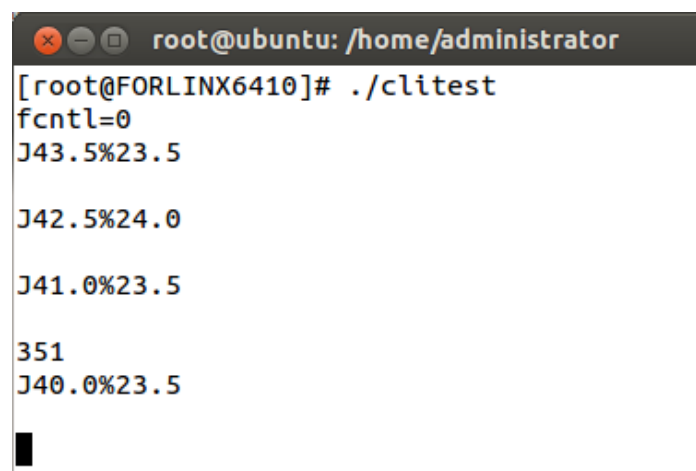

Fig.5 Monitoring interface

It shows that the user receives the real-time data correctly, and the data processing center can decode the commands correctly.

\section{Conclusions}

In the mode of using browser to monitor, as long as the mobile phone or computer connected to the Internet, the user can monitor the greenhouse conveniently. And because the user do not have to install application program in each control terminal, its application and popularization has higher flexibility.

\section{References}

[1] Wang Hui,Wang Shengwei,Wang Zhiqiang.The Mobile Intelligent Control System of Greenhouse Based on IoT and WiFi[J].Agricultural Mechanization Research,2014,04:187-190.

[2] Salleh A,Aziz A,Abidin M Z,et al.Development of greenhouse monitoring using wireless sensor network through Zigbee technology [J].International Journal of Engineering Science Invention,2013,2(7):6-12

[3] Li Pingping,Wang Jizhang.Reaserch Progress of Intelligent Management for Greenhouse Environment Information[J].Journal of Agricultural Machinery,2014,06:.

[4] Zeng Huan, Liu Yi.Application of Embedded WiFi Technology Used in the Gr eenhouse Environment Monitoring System[J],Forestry Machinery and Woodworking Equipment , 2008,02:49-51.

[5] YuanHongbo, ZhangDening, WenPeng.Environment Detection System of Agricultural Greenhouse Basedon GPRS.Agricultural Mechanization Research,2011,05:123-125+129.

[6] Liu Honggang.Application of Memory Database SQLite in Monitoring System[J].Hebei Electric Power Technology,2014,03:29-31. 